\title{
Carboxypeptidase E Is a Novel Modulator of RANKL-Induced Osteoclast Differentiation
}

\author{
Hyun-Ju Kim ${ }^{1,2, *}$, JungMin Hong ${ }^{2}$, Hye-Jin Yoon ${ }^{2}$, Young-Ran Yoon ${ }^{1}$, and Shin-Yoon Kim ${ }^{2,3, *}$
}

\begin{abstract}
Osteoclasts are large polykaryons that have the unique capacity to degrade bone and are generated by the differentiation of myeloid lineage progenitors. To identify the genes involved in osteoclast development, we performed microarray analysis, and we found that carboxypeptidase $E$ (CPE), a prohormone processing enzyme, was highly upregulated in osteoclasts compared with their precursors, bone marrow-derived macrophages (BMMs). Here, we demonstrate a novel role for CPE in receptor activator of NF-kB ligand (RANKL)-induced osteoclast differentiation. The overexpression of CPE in BMMs increases the formation of tartrate-resistant acid phosphatase (TRAP)-positive multinuclear osteoclasts and the expression of c-Fos and nuclear factor of activated T cells c1 (NFATc1), which are key regulators in osteoclastogenesis. Furthermore, employing CPE knockout mice, we show that CPE deficiency attenuates osteoclast formation. Together, our data suggest that CPE might be an important modulator of RANKL-induced osteoclast differentiation.
\end{abstract}

\section{INTRODUCTION}

Bone homeostasis is maintained by the balance between boneresorbing osteoclasts and bone-forming osteoblasts during bone remodeling (Anderson et al., 1997; Lacey et al., 1998). Excessive increases in osteoclastic bone resorption generally cause bone diseases such as osteoporosis and rheumatoid arthritis, and metastatic cancers (Baron and Hesse, 2012; Boyle et al., 2003). Osteoclasts are multinucleated cells that play a specialized role in bone resorption. These polykaryons differentiate from hematopoietic precursors of the monocyte/macrophage lineage in the presence of receptor activator of NF-кB ligand (RANKL) and macrophage colony-stimulating factor (M-

${ }^{1}$ Department of Molecular Medicine, Cell and Matrix Research Institute, Clinical Trial Center, School of Medicine,Kyungpook National University, Daegu 700-422, Korea, ${ }^{2}$ Skeletal Diseases Genome Research Center, Kyungpook National University Hospital, Daegu 700-412, Korea ${ }^{3}$ Department of Orthopedic Surgery, School of Medicine, Kyungpook National University, Daegu 700-422, Korea

*Correspondence: biohjk@hanmail.net(HJK); syukim@knu.ac.kr (SYK)

Received 26 June, 2014; revised 29 July, 2014; accepted 30 July, 2014; published online 15 September, 2014

Keywords: c-Fos, CPE, NFATc1, osteoclast, RANKL
CSF)(Novack and Teitelbaum, 2008; Teitelbaum, 2007; Teitelbaum and Ross, 2003).

Binding of RANKL to its receptor, RANK, which is expressed on osteoclast precursor cells, induces the recruitment of tumor necrosis factor receptor-associated factor (TRAF) molecules. RANKL binding to RANK triggers signal transduction processes involving NF- $\mathrm{KB}$ and mitogen-activated protein kinases. The activation of RANKL/RANK system leads to the induction of cFos and nuclear factor of activated T cells c1 (NFATc1), which are crucial transcription factors required for osteoclast differentiation. NFATc1, in turn, regulates the expression of osteoclastspecific genes, including tartrate-resistant acid phosphatase (TRAP) and cathepsin K.

Carboxypeptidase $\mathrm{E}$ (CPE) is a prohormone processing peptidase that is abundant in brain and endocrine tissues (Fricker and Snyder, 1982; Hook and Loh, 1984). This enzyme cleaves basic residues from the carboxy-terminal end of neuropeptide intermediates to yield mature bioactive forms (Cawley et al., 2012). CPE has also been shown to act as a prohormone sorting receptor for the regulated secretory pathway in endocrine and neuroendocrine cells (Cool et al., 1997). In addition, CPE is implicated in physiological cellular function and many diseases such as diabetes (Cawley et al., 2004; Leiter et al., 1999; Naggert et al., 1995), obesity (Cawley et al., 2004; Leiter et al., 1999), and tumor growth and metastasis (Du et al., 2001; He et al., 2004; Lee et al., 2011; Murthy et al., 2010; Tang et al., 2009), suggesting that CPE is a potential therapeutic drug target. Interestingly, previous studies have suggested a role for CPE in skeletal development. These studies demonstrated abundant expression of CPE in developing skeletal structures (Zheng et al., 1994) and growth plate chondrocytes (Zhang et al., 2008), although its specific role was not determined in these studies. Recently, Cawley et al. (2010) reported that CPE indirectly regulates bone metabolism through the sympathetic nervous system. However, the direct role of CPE in osteoclast differentiation is still poorly understood.

In this study, we identify CPE as a novel osteoclastogenic protein that is upregulated during osteoclast differentiation. We further reveal that CPE has a direct role in osteoclast differentiation induced by RANKL.

\section{MATERIALS AND METHODS}

\section{Animals}

Heterozygous $\left(\mathrm{Cpe}^{+-}\right)$mice were obtained from The Jackson Laboratory (USA). Mice were bred in our animal facility. Geno- 
The Role of CPE in Osteoclastogenesis

Hyun-Ju Kim et al.

typing was performed according to the protocol supplied by The Jackson Laboratory. All experimental procedures were performed with the approval of the Committee on the Ethics of Animal Experiments of the Kyungpook National University(Approval No. KNU-2011-99).

\section{Reagents}

RANKL and M-CSF were obtained from R\&D Systems (USA). Antibodies against CPE, NFATc1, and cathepsinK were purchased from BD Biosciences (USA), BD Pharmingen (USA), and Millipore (USA), respectively.Antibody for c-Fos was purchased from Santa Cruz Biotechnology (USA) and $\beta$-actin was obtained from Sigma-Aldrich(USA).

\section{Osteoclast culture}

Bone marrow-derived macrophages (BMMs) wereprepared from bone marrow cells as described previously (Kim et al., 2012). To generate osteoclasts, BMMs were cultured for 4 days with $\alpha$ minimal essential medium ( $\alpha$-MEM) containing 10\% fetal bovine serum (FBS) in the presence of RANKL $(20 \mathrm{ng} / \mathrm{ml})$ and M-CSF $(10 \mathrm{ng} / \mathrm{ml})$. The media were changed every 2 days.

\section{Tartrate-resistant acid phosphatase (TRAP) staining}

Cells were fixed in 4\% paraformaldehyde for $20 \mathrm{~min}$ and subsequently stained for TRAP activity with a $0.1 \mathrm{M}$ acetate solution $(\mathrm{pH}$ 5.0) containing $6.76 \mathrm{mM}$ sodium tartrate, $0.1 \mathrm{mg} / \mathrm{ml}$ naphthol ASMX phosphate, and $0.5 \mathrm{mg} / \mathrm{ml}$ Fast Red Violet. TRAP-positive multinucleated cells with three or more nuclei were scored.

\section{TRAP solution assay (TRAP activity)}

To measure TRAP activity, cells were fixed and incubated with 70 $\mu \mathrm{l}$ (in a 96 well plate) of citrate buffer $(50 \mathrm{mM}, \mathrm{pH} 4.6)$ containing $10 \mathrm{mM}$ sodium tartrate and $10 \mathrm{mM}$ p-nitrophenylphosphate (Sigma-Aldrich) for $15 \mathrm{~min}$ at $37^{\circ} \mathrm{C}$. The enzyme reaction mixtures were then transferred into new plates containing an equal volume of $0.1 \mathrm{~N} \mathrm{NaOH}$. Absorbance was measured at $405 \mathrm{~nm}$ using a microplate reader.

\section{Retroviral transduction}

CPE was cloned into the $\mathrm{PMX}$ retroviral vector and transfected into Plat-E packaging cells (Morita et al., 2000)using TransIT-LT1 (Mirus, USA). The viral supernatant was collected from the culture media 24 to $48 \mathrm{~h}$ after transfection. BMMs were infected with the virus for $24 \mathrm{~h}$ in the presence of $4 \mu \mathrm{g} / \mathrm{ml}$ polybrene (Sigma-Aldrich). The cells were selected with $1 \mu \mathrm{g} / \mathrm{ml}$ blasticidin for 3 days and subsequently used for osteoclast generation.

Reverse transcription polymerase chain reaction (RT-PCR) RT-PCR was performed as described previously (Kim et al., 2012). The following primers were used: CPE, 5'-ATGGTAATG AGGCGGTTGG-3' and 5'-TTCTTGCGACAGGGAGGT-3'; TRAP, 5'-ACAGCCCCCCACTCCCACCCT-3' and 5'-TCAGGGTCTGGGTCTCCTTGG-3'; cathepsin K, 5'-GGAAGAAGACTCACCAGAAGC-3' and 5'-GTCATATAGCCGCCTCCACAG-3'; RANK, 5'-TTTGTGGAATTGGGTCAATGAT-3' and 5'-ACCTCGCTGACCAGTGTGAA-3'; TRAF6, 5'-GCTCAAACGGACCATTCGGA-3' and 5'-GGGATTGTGGGTCGCTGAAA-3'; c-Fms, 5'-GAGCCTCTTGCAGGAGGTG-3' and 5'-GGTCCAATGGGCAGCTGG-3'; and GAPDH, 5'-ACTTTGTCAAGCTCATTTCC$3^{\prime}$ and 5'-TGCAGCGAACTTTATTGATG-3'.

\section{Real-time PCR}

Real-time PCR was performed using an ABI 7500 Real-Time PCR System and SYBR Green dye (Applied Biosystems, USA). The following primers were used: c-Fos, 5'-AGGCCCAGT-
GGCTCAGAGA-3' and 5'-GCTCCCAGTCTGCTGCATAGA-3'; NFATC1, 5'-ACCACCTTTCCGCAACCA-3' and 5'-TTCCGTTTCCCGTTGCA-3'; TRAP, 5'-TCCCCAATGCCCCATTC-3' and 5'-CGGTTCTGGCGATCTCTTTG-3'; cathepsin K, 5'-GGCTGTGGAGGCGGCTAT-3' and 5'-AGAGTCAATGCCTCCGTTCTG-3'.

\section{Western blotting}

Cultured cells were lysed in lysis buffer containing 50 mMTris (pH 7.4), $150 \mathrm{mMNaCl}, 1 \% \mathrm{NP}-40,1 \mathrm{mM}$ EDTA, and protease and phosphatase inhibitors. The protein concentration was determined using the Bicinchoninic Acid Kit (Pierce, USA), and equal amounts of proteins were separated on a sodium dodecyl sulfate (SDS)-polyacrylamide gel. The gels were blotted onto nitrocellulose membranes and incubated with primary antibodies. The immunoreactive proteins were detected with enhanced chemiluminescence reagents (ECL-plus, Amersham, GE Healthcare) after incubation with the appropriate secondary antibody.

\section{Immunofluorescence}

Cells were fixed with $4 \%$ paraformaldehyde for $20 \mathrm{~min}$ and permeabilized with $0.1 \%$ Triton X-100 for 10 min. After blocking with $1 \%$ bovine serum albumin for $10 \mathrm{~min}$, the cells were labeled with anti-CPE antibody (1:100) in blocking solution for $1 \mathrm{~h}$. The samples were rinsed with phosphate-buffered saline (PBS) and incubated with Alexa Fluor 488 goat anti-mouse IgG antibody (1:100, Invitrogen) for $1 \mathrm{~h}$. After washing with PBS, the samples were mounted with $90 \%$ glycerol in PBS and observed using a fluorescence microscope (Nikon, Japan).

\section{Statistical analysis}

All experiments were performed in triplicate. The data are presented as the means \pm S.D. Statistical significance was determined using a two-tailed Student's $t$-test.

\section{RESULTS}

CPE expression is upregulated during osteoclastogenesis To identify genes that are regulated during osteoclast development, we performed microarray analysis and compared the gene expression profiles between undifferentiated precursor cells and fully differentiated osteoclasts. The microarray data showed that CPE expression was increased by 43-fold in osteoclasts compared with primary bone marrow-derived macrophages (BMMs) (data not shown). To confirm the relevance of this observation, we examined the expression pattern of CPE during RANKL-induced osteoclast differentiation. BMMs were isolated and subsequently cultured in the presence of osteoclastogenic cytokines. We found that CPE mRNA expression was timedependently elevated during osteoclastogenesis (Fig. 1A). Similarly, the protein level of CPE was strongly increased as the cells differentiated (Fig. 1B). This pattern of CPE protein expression was further confirmed by immunofluorescent staining. We observed clear staining of CPE in mature osteoclasts; however, we detected very low CPE signals in undifferentiated BMMs (Fig. 1C).

Overexpression of CPE enhances osteoclast formation The expression pattern of CPE suggests that CPE may have biological significance in the context of osteoclast development. To examine this possibility, we retrovirally transduced primaryBMMs with CPE or control (pMX empty vector). The overexpression of CPE was confirmed by RT-PCR (Fig. 2A).Transduced BMMs were cultured in the presence of M-CSF and various concentrations of RANKL for 4 days. The cells were then stained for TRAP, which is a cytochemical marker of osteoclasts. The overexpression of CPE significantly increased the number of TRAP- positive multi- 
A

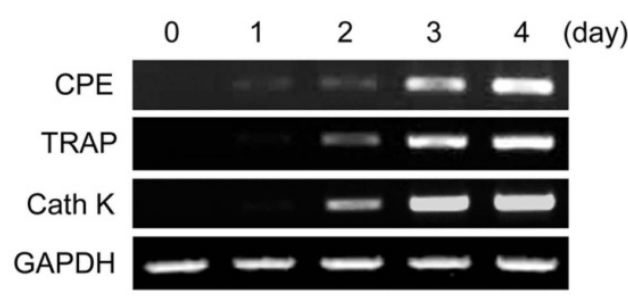

B

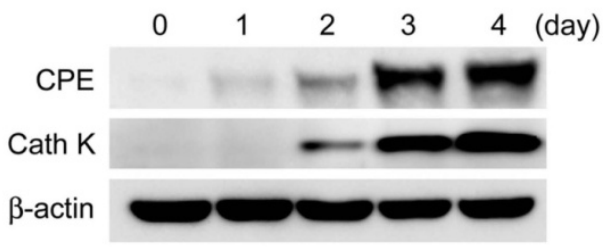

C

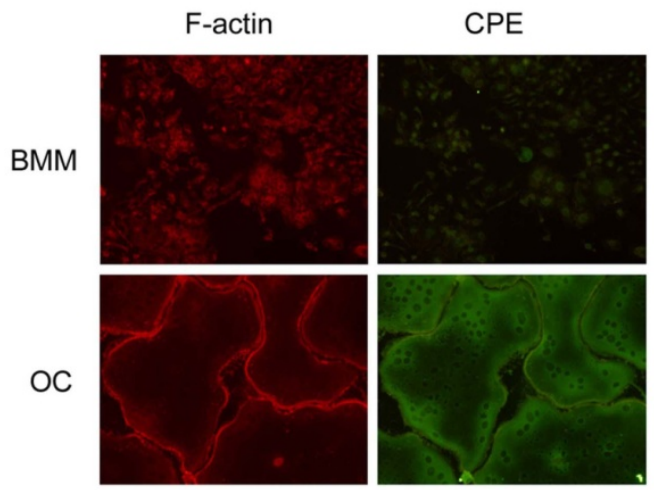

Fig. 1. Expression of CPE is enhanced during osteoclast differentiation. BMMs were cultured in M-CSF $(10 \mathrm{ng} / \mathrm{ml})$ and RANKL (20 $\mathrm{ng} / \mathrm{ml}$ ) for the indicated number of days. (A) Expression of CPE was determined by RT-PCR. (B) CPE expression was analyzed by immunoblotting. TRAP or cathepsinK (CathK) served as positive controls for osteoclastogenesis, and GAPDH or $\beta$-actin served as loading controls. (C) BMMs or osteoclasts (OC) were fixed and permeabilized prior to staining. F-actin and CPE were stained with TRITC-conjugated phalloidin (red) and anti-CPE antibody (green), respectively.

nuclear osteoclasts (MNCs) at all concentrations of RANKL (Figs. $2 \mathrm{~B}$ and $2 \mathrm{C}$ ). In agreement with the increased numbers of TRAPexpressing cells, TRAP activity, as measured by TRAP solution assay, was also elevated in CPE-transduced osteoclasts (Fig. 2D). We also observed that the overexpression of CPE did not affect precursor proliferation or survival by means of an MTS assay (data not shown).

Overexpression of CPE enhances the induction of c-Fos and NFATc1

Because ectopic expression of CPE increased osteoclast formation, we next examined the impact of CPE overexpression onthe expression of osteoclastogenic markers. As shown in Fig. $3 A$, the mRNA expression of c-Fos and NFATc1, key transcriptionfactors for osteoclastogenesis, was significantly increased in CPE-transduced osteoclasts compared with vector-infected cells.Reflecting the enhanced NFATc1 expression, the mRNA expression of NFATc1 target genes, TRAP and cathepsinK, was also increased by the overexpression of CPE (Fig. 3A). This
A

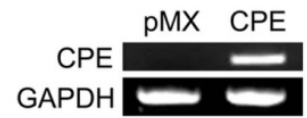

B

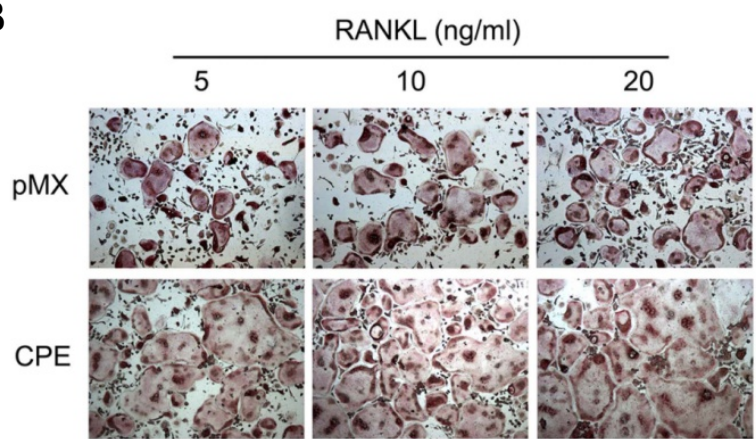

C

D
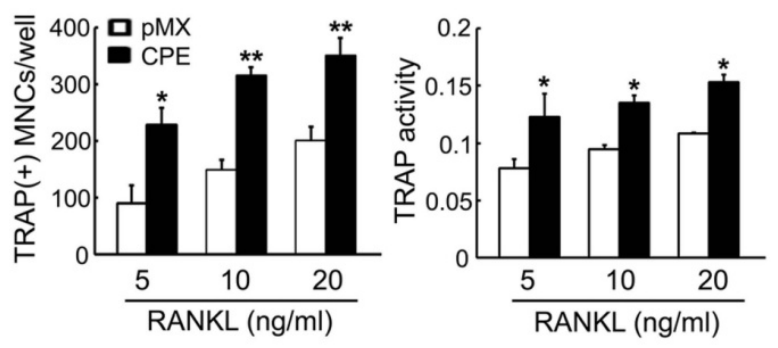

Fig. 2. Overexpression of $\mathrm{CPE}$ enhances osteoclast formation. BMMs were transduced with $\mathrm{pMX}$ vector (control) or CPE (pMX-CPE) retrovirus. (A) CPE expression was analyzed by RT-PCR. (B) BMMs were cultured with M-CSF $(10 \mathrm{ng} / \mathrm{ml})$ and the indicated concentrations of RANKL and stained for TRAP after 4 days. (C) The TRAPpositive multinucleated cells (MNCs) were counted. (D) TRAP activity was determined by measuring the OD values at $405 \mathrm{~nm}$ on day 4 . The data are expressed as the means $\pm \mathrm{SD}$. ${ }^{\star} P<0.05$, ${ }^{\star \star} P<0.001$.

CPE-mediated upregulation of c-Fos, NFATc1, and cathepsinK was further confirmed by immunoblotting analysis (Fig. 3B). On the other hand, the overexpression of CPE did not affect the mRNA expression of RANK, c-Fms, or TRAF6 in response to RANKL (Fig. 3C). Overall, these results indicate that CPE overexpression enhances RANKL-induced osteoclast differentiation.

Deficiency of CPE attenuates osteoclast formation

We next investigated the physiologic role of CPE in osteoclast differentiation. BMMs from WT or CPE knockout (KO) mice were cultured with M-CSF and two different concentrations of RANKL. In both concentrations of RANKL, the deficiency of CPE attenuated osteoclast formation (Figs. 4A and 4B). Reflecting the decreased numbers of TRAP-positive cells, TRAP activity wasalso reduced in CPE KO mice compared with WT mice (Fig. 4C). These results indicate that CPE acts as a positive modulator in RANKL-mediated osteoclastogenesis.

\section{DISCUSSION}

Osteoclast development is a sequential multistep event involvinghematopoietic progenitor generation, differentiation, fusion, andactivation. This process requires several biomolecules; however, many molecules involved in osteoclast development remain unidentified. To identify genes regulated by RANKL, we per- 
A

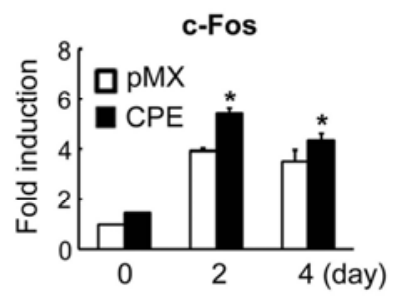

C

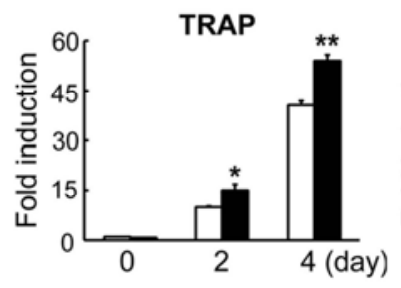

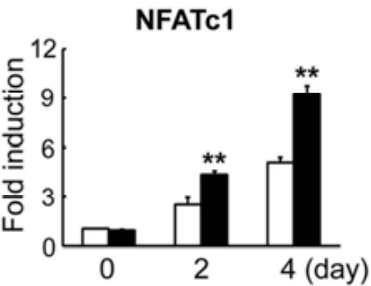

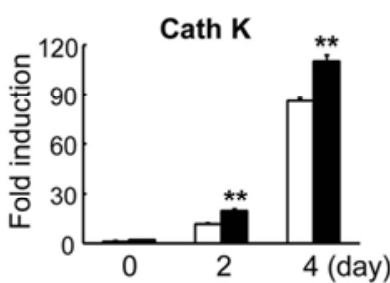

B
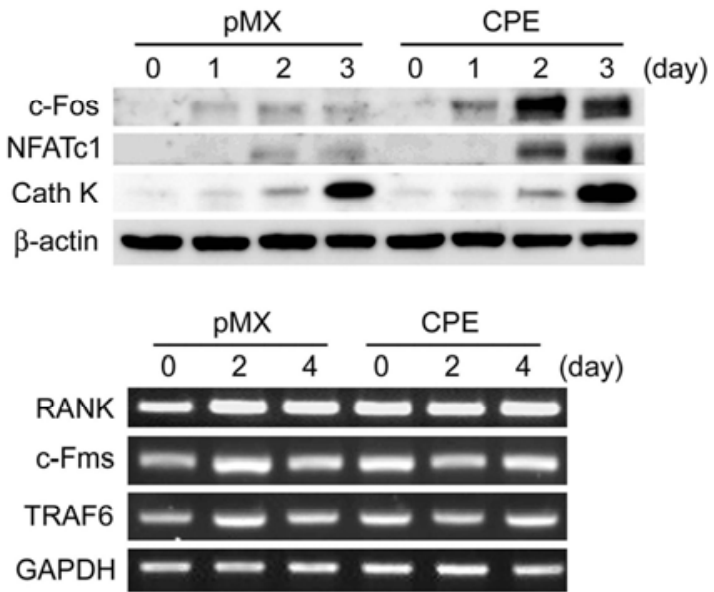

Fig. 3. Overexpression of CPE increases the expression of c-Fos and NFATc1. BMMs were transduced with pMX control or CPE retrovirus and cultured with M-CSF (10 ng/ml) and RANKL (20 ng/ml) for the indicated number of days. Real-time PCR (A), immunoblotting (B), or RT-PCR (C) was performed to assess the expression of the indicated genes. GAPDH and $\beta$-actin served as loading controls. The data are expressed as the means \pm SD. ${ }^{\star} P<0.05,{ }^{\star \star} P<0.001$.

A

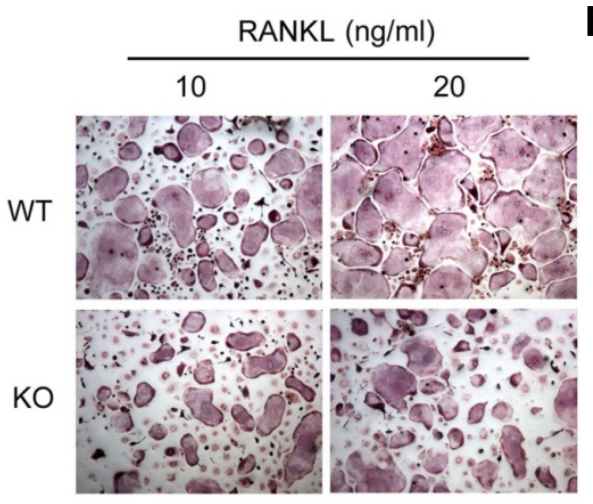

B

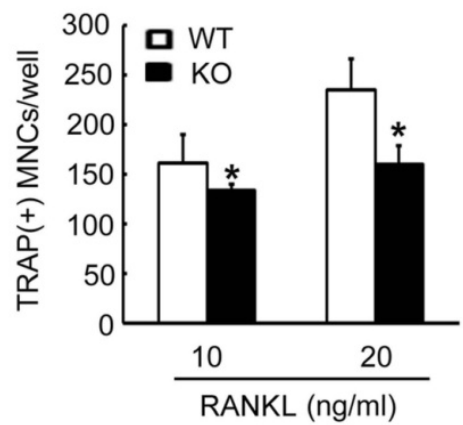

C

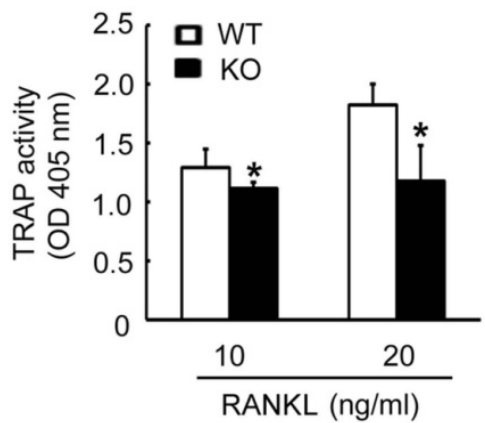

Fig. 4. Deficiency of CPE decreases osteoclast formation. BMMs derived from WT or CPE KO mice were cultured for 4 days with $10 \mathrm{ng} / \mathrm{ml}$ of $\mathrm{M}$ CSF and the indicated concentrations of RANKL. (A) Cells were fixed and stained for TRAP. (B) Numbers of TRAP-positive MNCs were counted. (C) TRAP activity was determined. The data are expressed as the means \pm SD. ${ }^{*} P<0.05$.

formed microarray analysis using primary BMMs after stimulation with RANKL. We observed that CPE expression was abundant in bone-resorbing osteoclasts. The expression of CPE was very low in undifferentiated progenitor cells and was increased during the osteoclastogenic process. The expression pattern of CPE implies that CPE may have an important role in osteoclast development. Indeed, we found that CPE positively regulates osteoclast differentiation.

Previous studies identified CPE as a highly expressed gene in the context of skeletal development. Studies utilizing in situ hybridization during development revealed that CPE was expressed in cartilage primordium in cephalic bones (Zheng et al., 1994). In addition, microarray analysis of perichondral and reserve chondrocytes in the growth plates of long bones demonstrated that CPE was abundant in both zones (Zhang et al., 2008). These studies proposed that CPE may be involved in skeletal development. In the current study, we provide clear evidence for the expression of CPE in osteoclast lineage cells and its role in osteoclast differentiation.
It has been reported that CPE knockout $(\mathrm{KO})$ mice, an obese animal model, have low bone mineral density (Cawley et al., 2010). The study demonstrated that CPE KO mice had reduced levels of the hypothalamic neuropeptides, resulting in an overall increase in the ratio of RANKL/osteoprotegerin. Although this study highlighted the importance of CPE in energy metabolism and bone remodeling, the direct role of CPE in osteoclast differentiation was not investigated. On the other hand, our study shows that CPE directly regulates RANKL-induced osteoclastogenesis. Furthermore, our work also reveals that the deficiency of CPE results in decreased osteoclast formation. Together, both studies clearly indicate that CPE is important for bone metabolism and remodeling.

c-Fos and NFATC1 are key transcription factors that regulate the induction of osteoclastogenic genes. Mice that are deficient in c-Fos exhibit severe osteopetrotic phenotype due to defective osteoclast formation (Johnson et al., 1992; Wang et al., 1992). The important role of NFATc1 in osteoclastogenesis was demonstrated by in vitro experiments. Embryonic stem cells 
derived from NFATc1-deficient mice cannot differentiate into osteoclasts, and ectopic expression of NFATc1 results in the generation of osteoclasts from precursor cells in the absence of RANKL (Hirotani et al., 2004; Takayanagi et al., 2002). In vivo expression of constitutively active NFATc1 results in osteopenia due to increased osteoclast differentiation (Ikeda et al., 2006). In this study, we found that the overexpression of CPE increases the expression of both c-Fos and NFATC1. Thus, critical for the acceleration of osteoclast differentiation. Further studies will be necessary to clarify the molecular mechanism underlying CPEmediated induction of these transcription factors by RANKL.

CPE cleaves hormone precursors, including proopiomelanocortin (POMC), and produces several physiologically important peptides (D'Agostino and Diano, 2010). It has been demonstrated that POMC is expressed in osteoclasts (Zhong et al., 2005), suggesting that a POMC-derived peptide may have a direct role in osteoclast development. In agreement with these observations, we also found that POMC was expressed in osteoclast lineage cells (data not shown). One POMC-derived hormone often studied in skeletal cells is alpha-melanocyte stimulating hormone $(\alpha-\mathrm{MSH})$. Cornish et al. (2003) reported the direct effect of $\alpha-\mathrm{MSH}$ on osteoclasts, showing that $\alpha-\mathrm{MSH}$ stimulates osteoclast formation from BMMs and administration of $\alpha$ $\mathrm{MSH}$ to mice decreased bone volume and trabecular number. In this regard, increased levels of $\alpha-\mathrm{MSH}$ by CPE overexpression would likely to contribute to CPE-mediated enhancement of osteoclast formation.

In summary, we report that the metallocarboxypeptidase family member CPE is upregulated during osteoclast development. Our results demonstrate that the overexpression of CPE increases osteoclast differentiation through the induction of c-Fos and NFATC1, key regulators of osteoclastogenesis. Furthermore, we show that CPE deficiency decreases osteoclast generation in response to RANKL. Thus, in addition to its indirect effects on bone metabolism through the regulation of peptide hormone processing, CPE exerts direct effects on osteoclasts and plays a pivotal role in osteoclast differentiation.

\section{ACKNOWLEDGMENTS}

This research was supported by the Basic Science Research Program of the National Research Foundation of Korea (NRF) funded by the Ministry of Education, Science, and Technology (2013R1A1A2A10005515).

\section{REFERENCES}

Anderson, D.M., Maraskovsky, E., Billingsley, W.L., Dougall, W.C., Tometsko, M.E., Roux, E.R., Teepe, M.C., DuBose, R.F., Cosman, D., and Galibert, L. (1997). A homologue of the TNF receptor and its ligand enhance T-cell growth and dendritic-cell function. Nature 390, 175-179.

Baron, R., and Hesse, E. (2012). Update on bone anabolics in osteoporosis treatment: rationale, current status, and perspectives. J. Clin. Endocrinol. Metabol. 97, 311-325.

Boyle, W.J., Simonet, W.S., and Lacey, D.L. (2003). Osteoclast differentiation and activation. Nature 423, 337-342.

Cawley, N.X., Zhou, J., Hill, J.M., Abebe, D., Romboz, S., Yanik, T., Rodriguiz, R.M., Wetsel, W.C., and Loh, Y.P. (2004). The carboxypetidase E Knockout mouse exhibits endocrinological and behavioral deficits. Endocrinology 145, 5807-5819.

Cawley, N.X., Yanik, T., Woronowicz, A., Chang, W., Marini, J.C., and Loh, Y.P. (2010). Obese carboxypeptidase E knockout mice exhibit multiple defects in peptide hormone processing contributing to low bone mineral density. Am. J. Physiol. Endocrinol. Metab. 299, E189-197.

Cawley, N.X., Wetsel, W.C., Murthy, S.R., Park, J.J., Pacak, K., and Loh, Y.P. (2012). New roles of carboxypeptidase $E$ in endocrine and neural function and cancer. Endocrine Rev. 33, 216-253.
Cool, D.R., Normant, E., Shen, F., Chen, H.C., Pannell, L., Zhang, Y., and Loh, Y.P. (1997). Carboxypeptidase $E$ is a regulated secretory pathway sorting receptor: genetic obliteration leads to endocrine disorders in Cpe(fat) mice. Cell 88, 73-83.

Cornish, J., Callon, K.E., Mountjoy, K.G., Bava, U., Lin, J.M., Myers, D.E., Naot, D., and Reid, I.R. (2003). alpha -melanocytestimulating hormone is a novel regulator of bone. American journal of physiology. Endocrinol. Metabol. 284, E1181-1190.

D’Agostino, G., and Diano, S. (2010). Alpha-melanocyte stimulating hormone: production and degradation. J. Mol. Med. 88, 11951201.

Du, J., Keegan, B.P., and North, W.G. (2001). Key peptide processing enzymes are expressed by breast cancer cells. Cancer Lett. 165, 211-218.

Fricker, L.D., and Snyder, S.H. (1982). Enkephalin convertase: purification and characterization of a specific enkephalin-synthesizing carboxypeptidase localized to adrenal chromaffin granules. Proc. Natl. Acad. Sci. USA 79, 3886-3890.

He, P., Varticovski, L., Bowman, E.D., Fukuoka, J., Welsh, J.A., Miura, K., Jen, J., Gabrielson, E., Brambilla, E., Travis, W.D., et al. (2004). Identification of carboxypeptidase $\mathrm{E}$ and gamma-glutamyl hydrolase as biomarkers for pulmonary neuroendocrine tumors by cDNA microarray. Hum. Pathol. 35, 1196-1209.

Hirotani, H., Tuohy, N.A., Woo, J.T., Stern, P.H., and Clipstone, N.A. (2004). The calcineurin/nuclear factor of activated T cells signaling pathway regulates osteoclastogenesis in RAW264.7 cells. J. Biol. Chem. 279, 13984-13992.

Hook, V.Y., and Loh, Y.P. (1984). Carboxypeptidase B-like converting enzyme activity in secretory granules of rat pituitary. Proc. Natl. Acad. Sci. USA 81, 2776-2780.

Ikeda, F., Nishimura, R., Matsubara, T., Hata, K., Reddy, S.V., and Yoneda, T. (2006). Activation of NFAT signal in vivo leads to osteopenia associated with increased osteoclastogenesis and boneresorbing activity. J. Immunol. 177, 2384-2390.

Johnson, R.S., Spiegelman, B.M., and Papaioannou, V. (1992). Pleiotropic effects of a null mutation in the c-fos proto-oncogene. Cell 71, 577-586.

Kim, H.J., Hong, J.M., Yoon, K.A., Kim, N., Cho, D.W., Choi, J.Y., Lee I.K., and Kim, S.Y. (2012). Early growth response 2 negatively modulates osteoclast differentiation through upregulation of Id helix-loop-helix proteins. Bone 51, 643-650.

Lacey, D.L., Timms, E., Tan, H.L., Kelley, M.J., Dunstan, C.R., Burgess, T., Elliott, R., Colombero, A., Elliott, G., Scully, S., et al. (1998). Osteoprotegerin ligand is a cytokine that regulates osteoclast differentiation and activation. Cell 93, 165-176.

Lee, T.K., Murthy, S.R., Cawley, N.X., Dhanvantari, S., Hewitt, S.M., Lou, H., Lau, T., Ma, S., Huynh, T., Wesley, R.A., et al. (2011). An $\mathrm{N}$-terminal truncated carboxypeptidase $\mathrm{E}$ splice isoform induces tumor growth and is a biomarker for predicting future metastasis in human cancers. J. Clin. Invest. 121, 880-892.

Leiter, E.H., Kintner, J., Flurkey, K., Beamer, W.G., and Naggert, J.K. (1999). Physiologic and endocrinologic characterization of male sex-biased diabetes in C57BLKS/J mice congenic for the fat mutation at the carboxypeptidease E locus. Endocrine 10, 57-66.

Morita, S., Kojima, T., and Kitamura, T. (2000). Plat-E: an efficient and stable system for transient packaging of retroviruses. Gene Ther. 7, 1063-1066.

Murthy, S.R., Pacak, K., and Loh, Y.P. (2010). Carboxypeptidase E: elevated expression correlated with tumor growth and metastasis in pheochromocytomas and other cancers. Cell. Mol. Neurobiol. 30, 1377-1381.

Naggert, J.K., Fricker, L.D., Varlamov, O., Nishina, P.M., Rouille, Y., Steiner, D.F., Carroll, R.J., Paigen, B.J., and Leiter, E.H. (1995). Hyperproinsulinaemia in obese fat/fat mice associated with a carboxypeptidase E mutation which reduces enzyme activity. Nat. Genet. 10, 135-142.

Novack, D.V., and Teitelbaum, S.L. (2008). The osteoclast: friend or foe? Ann. Rev. Pathol. 3, 457-484.

Takayanagi, H., Kim, S., Koga, T., Nishina, H., Isshiki, M., Yoshida, H. Saiura, A., Isobe, M., Yokochi, T., Inoue, J., et al. (2002). Induction and activation of the transcription factor NFATc1 (NFAT2) integrate RANKL signaling in terminal differentiation of osteoclasts. Dev. Cell 3, 889-901.

Tang, S.S., Zhang, J.H., Liu, H.X., and Li, H.Z. (2009). PC2/CPEmediated pro-protein processing in tumor cells and its differentiated cells or tissues. Mol. Cell. Endocrinol. 303, 43-49. 
Teitelbaum, S.L. (2007). Osteoclasts: what do they do and how do they do it? Am. J. Pathol. 170, 427-435.

Teitelbaum, S.L., and Ross, F.P. (2003). Genetic regulation of osteoclast development and function. Nat. Rev. Genet. 4, 638-649.

Wang, Z.Q., Ovitt, C., Grigoriadis, A.E., Mohle-Steinlein, U., Ruther,U., and Wagner, E.F. (1992). Bone and haematopoietic defects in mice lacking c-fos. Nature 360, 741-745.

Zhang, M., Pritchard, M.R., Middleton, F.A., Horton, J.A., and Damron, T.A. (2008). Microarray analysis of perichondral and reserve growth plate zones identifies differential gene expressions and signal pathways. Bone 43, 511-520.

Zheng, M., Streck, R.D., Scott, R.E., Seidah, N.G., and Pintar, J.E. (1994). The developmental expression in rat of proteases furin PC1, PC2, and carboxypeptidase E: implications for early maturation of proteolytic processing capacity. J. Neurosci.14, 46564673.

Zhong, Q., Sridhar, S., Ruan, L., Ding, K.H., Xie, D., Insogna, K., Kang, B., Xu, J., Bollag, R.J., and Isales, C.M. (2005). Multiple melanocortin receptors are expressed in bone cells. Bone 36, 820-831. 